\title{
Generative Adversarial Neural Networks and Deep Learning: Successful Cases and Advanced Approaches
}

\author{
OLEKSANDR STRIUK, YURIY KONDRATENKO \\ Petro Mohyla Black Sea National University, 10 68th Desantnykiv st., Mykolaiv, 54003, Ukraine \\ (e-mail: oleksandr.striuk@gmail.com,y_kondrat2002@yahoo.com) \\ Corresponding author: Oleksandr Striuk (e-mail: oleksandr.striuk@gmail.com).
}

\begin{abstract}
$\therefore$ ABSTRACT Cross-domain artificial intelligence (AI) frameworks are the keys to amplify progress in science. Cutting edge deep learning methods offer novel opportunities for retrieving, optimizing, and improving different data types. AI techniques provide new ways for enhancing and polishing existing models that are used in applied sciences. New breakthroughs in generative adversarial neural networks (GANNs/GANs) and deep learning allow to drastically increase the quality of diverse graphic samples obtained with research equipment. All these innovative approaches can be compounded into a unified academic and technological pipeline that can radically elevate and accelerate scientific research and development. The authors analyze a number of successful cases of GAN and deep learning applications in applied scientific fields (including observational astronomy, health care, materials science, deep fakes, bioinformatics, and typography) and discuss advanced approaches for increasing GAN and DL efficiency in terms of performance calibration using modified data samples, algorithmic enhancements, and various hybrid methods of optimization.
\end{abstract}

KEYWORDS generative adversarial network; neural networks; deep learning; machine learning; artificial intelligence.

\section{INTRODUCTION}

CURRENT artificial intelligence technologies such as deep learning (DL) and artificial neural networks - AI systems inspired by the structure and principles of the human brain - become true amplifiers of scientific discovery and development. AI helps to speed up experimental simulations, gather and process new data, prove brand new theoretical hypotheses in many scientific fields. AI is literally relevant to any intellectual task [1].

Deep learning is one of the machine learning methods that is grounded on an artificial neural networks framework that can be trained based on supervised and unsupervised learning algorithms. Deep learning architectures are effectively used in different fields including autonomous vehicles, computer vision, natural language processing, recommendation services, bioinformatics, medical image analysis, and generation of new functional samples, where they have shown similar to human experts' results or even outperformed them. The main concept of artificial neural networks was inspired by real biological systems. Generative adversarial network (GAN) is the implementation of a deep/machine unsupervised learning algorithm class that represents the architecture of two artificial neural networks that compete with each other in a zero-sum game.

Today's AI methods show incredibly successful practical results at doing science [2-6]. AI systems are used as an effective mechanism in diverse scientific fields transforming conventional research practices and expediting discoveries. The main advantage of $\mathrm{AI}$ is that it can outperform humans when it comes to processing large amounts of data, detecting patterns and abnormalities that human experts could never have spotted.

Fig. 1 demonstrates the integrated liaisons between key elements of AI. 


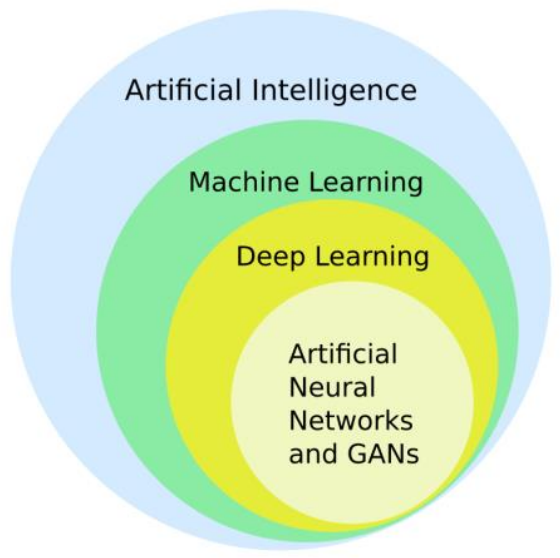

Figure 1. Interconnections and nesting of artificial intelligence systems.

The driving force that triggered an increased interest in the more intense integration of $\mathrm{AI}$ into science were massive arrays of data accumulated over many years of research and the development of high-performance computing platforms that were able to process and analyze these data sets. In recent years, artificial intelligence systems have made a great contribution to the intensification of scientific research.

\section{SCRUTINIZING HEURISTICS OF SUCCESSFUL CASES}

This paper is an overview analysis and considers the practical aspects of the use of machine learning and GAN in the applied fields of science. In particular, observational astronomy, health care, materials science and deep fake detection have been selected as illustrative examples.

Cases were selected from the most important areas that affect the core scientific research and quality of human life.

The next sections of the paper analyze the fragmented data of efficient research in this area and assess future prospects.

\section{A. GAN METHOD RECOVERS FEATURES IN ASTROPHYSICAL IMAGES OF GALAXIES}

Schawinski et al. demonstrated a machine learning method that was able to successfully recover elements in astronomical images of galaxies [7]. The said ML methodology allows to overcome the deconvolution limit using higher quality training data sets and makes it possible to reconstruct information from poor quality samples by successfully building priors [7].

The Nyquist-Shannon sampling theorem sets limitations in terms of removing the effect of the point spread function especially when there is noise, sequentially sampled material cannot be completely deconvolved without violating the theorem postulates $[7,8]$. As a workaround for this issue, Schawinski et al. applied a generative adversarial neural network (GANN/GAN).

A GAN is a state-of-the-art deep learning algorithm that allows two neural networks to contest with each other in the form of a zero-sum game. This framework can create realistic artificial graphical samples similar and almost identical to the images from a training set [9].

GAN works on the following principle: the first network, the generator, creates samples (candidates), and the second network, the discriminator, evaluates them, trying to distinguish real from fakes. The generative network tries to form a new sample by combining primary samples using latent space variables. The discriminator network learns to distinguish between real and counterfeit samples. Conventional deep learning models are used in GANs as components. For example, the discriminator can be implemented as a convolutional classifier network.

The suggested method can drastically improve the quality of obtained image samples of galaxies by recovering its properties and bypassing the deconvolution constraints mentioned above.

During the experiment the GAN has been trained on a data set that included 4,550 graphic samples of nearby galaxies in the redshift range $0.01<\mathrm{z}<0.02$ that were taken from the Sloan Digital Sky Survey. The results were assessed through ten cross-validation iterative cycles. GAN was able to restore artificially corrupted image samples with bad seeing and high noise levels (compared to the original image) and showed results that greatly outperform standard deconvolution.

The results proved the effectiveness of the method in restoring important characteristics of celestial phenomena and in expanding the range of study of existing astronomical data gathered by telescopes [7]. The images clearly show the ability of GAN to restore features that cannot be recovered using conventional deconvolution techniques.

Fig. 2 is a graphic illustration of the training process of the method described by Schawinski et al. A set of original images is the input. Image degradation is achieved by blurring, adding noise, and through convolution with a worse point spread function. Degraded images are automatically generated and used for GAN training. Only the generator is used for recovering images during the testing phase.

However, this technique is not without drawbacks and has its limitations. The main constraint is related to the limited capacity of the training set that drastically impacts the restorative capabilities of the method. Small training data results in a bad approximation. A model that is trained on such poor data will likely demonstrate low performance due to overfitting. As a possible solution, the training sets that consist of synthesized simulation images can be considered as an additional reinforcement technique in terms of learning. 
(a)

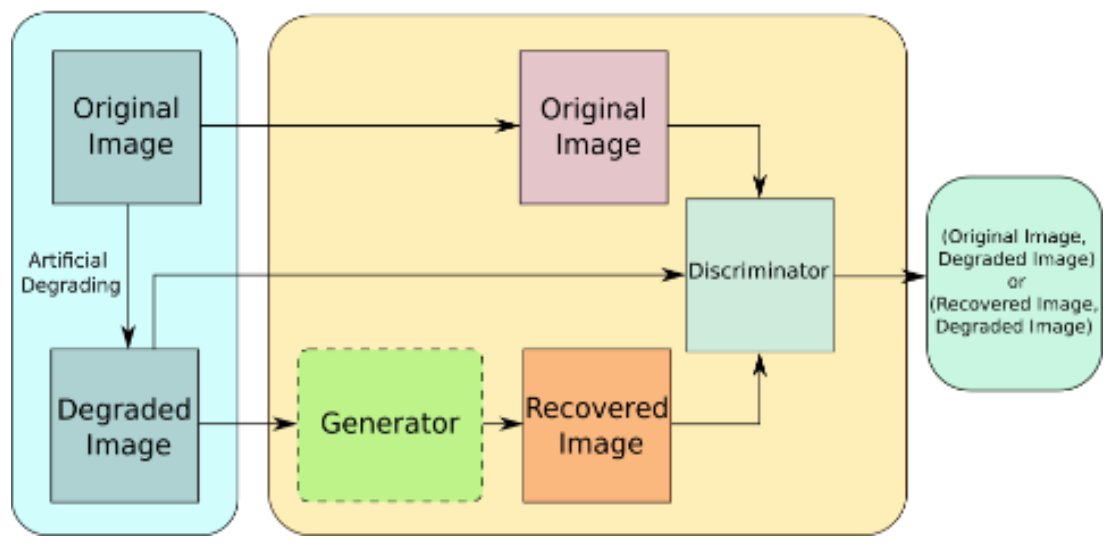

Figure 2. Graphic illustration of training process of GAN: (a) data preparation; (b) training of GAN

\section{B. DEEP LEARNING AND PHOTOMETRIC REDSHIFT ESTIMATION}

A. D’Isanto and K. L. Polsterer proposed a new experimental deep learning technique the aim of which was developing a new method for photometric redshift estimation [10]. The suggested approach demonstrates a novel technique for estimating PDF (probability density function) for redshifts based on imaging data in such a way that ultimately there is no need for additional steps of feature-extraction and featureselection. The PDF is presented by the following equation (1) $[10]$ :

$$
p(x)=\sum_{j=1}^{n} \omega_{j} \mathcal{N}\left(x \mid \mu_{j}, \sigma_{j}\right)
$$

where $\mathcal{N}\left(x \mid \mu_{j}, \sigma_{j}\right)$ is a normal distribution, $\mu_{j}$ is a given mean, $\sigma_{j}$ is standard deviation, $x$ is a given value, $\omega_{j}$ is a weighting factor of each component (all weights sum to one).

In order to achieve the objective, the researchers combined a deep convolutional network with a mixture density network. The evaluation was presented as Gaussian mixture models as a representation of the probability density functions in the redshift space. As an addition to the conventional estimation methods, the continuous ranked probability score (CRPS) and the probability integral transform (PIT) were implemented as performance criteria. The proposed method was capable of predicting redshift probability density functions regardless of the type of source (e.g., galaxies, stars, quasars) and showed better results that were performed by reference techniques and that were described in the scientific literature. This deep learning method is highly universal and is able to address any kind of probabilistic regression problem based on imaging data [10]. The reviewed example demonstrates the flexibility and versatility of artificial intelligence systems in astrophysical research related to the analysis of imaging data.

\section{GALAXY EVOLUTION RESEARCH WITH GENERATIVE MODELS}

Generative models demonstrate the potential for processing astronomical intelligence in a way that focuses on a datadriven approach. Kevin Schawinski, M. Dennis Turp, and Ce Zhang described a method that applies generative models to probe and research hypotheses in astrophysics and other scientific fields.

During the experiment, using a latent space representation of the data, the Fader artificial neural network has been trained to produce synthesized data for hypothesis verification [11].

The architecture of the Fader network is the implementation of the encoder-decoder system with a domain adversarial training element that implies researching and processing graphical samples in accordance with their physical features [12]. The Fader network tries to minimize the following objectives (given pairs of graphic samples and labels $\{x, y\})[12]$ :

$$
\begin{gathered}
\mathcal{L}_{a e}=-\frac{1}{m} \sum\|D(E(x), y)-x\|_{2}^{2}-\lambda_{E} \log (P(1- \\
y \mid E(x))), \\
\mathcal{L}_{\text {dis }}=-\frac{1}{m} \sum \log (P(y \mid E(x))),
\end{gathered}
$$

where $x$ represents images, $E(x)$ is the encoder (neural network) input, which is responsible for mapping from the image space to a latent representation of fixed dimension [12], $D(E(x), y)$ is the decoder (a neural network as well), it takes attempts to rebuild $x,\{x, y\}$ are binary labels, $\mathcal{L}_{a e}$ and $\mathcal{L}_{\text {dis }}$ are two loss functions that interact with each other through adversarial cooperation.

The quenching of star formation in galaxies was used as an illustration of the effectiveness of the method because this process is well described in astrophysical literature. In addition to approaches that are based on simulations and observations, this method can be useful in exploring important astronomical and other celestial phenomena from a different perspective [12]. The underlying reason why researchers picked this architecture is that the Fader can distinguish two data distributions and learn and visualize these differences.

As for the limitations of this approach, it is important to 
stress that the described method is mainly applicable to test hypotheses but not to prove them in a conventional way. Also, there is always room for mismatch when it comes to the collation of real data and imperfection of training sets and network design.

The proposed method requires domain knowledge management by the user since it is not completely unmanned. Anyway, the proposed method of applying Fader-like generative models in testing hypotheses and physical processes modeling showed noteworthy potential in astronomy and other scientific fields [12].

\section{D.4 SPATIAL-GANS AND SYNTHETIC IMAGING}

Michael J. Smith and James E. Geach focused their research endeavor on the problem of the small size of images produced by generative adversarial neural networks (GAN/GANN, which are mentioned above) and on the ability of the framework known as Spatial Generative Adversarial Networks (SGANs, designed and described by Jetchev et al.) to generate large graphic images, if training image samples demonstrate a certain level of periodicity isotropy (cosmological principle) makes deep imaging surveys fit the criterion $[13,14]$.

SGAN was trained to produce images resembling the eXtreme Deep Field (XDF) - the photo portrait of the universe that was assembled by combining 10 years of NASA Hubble Space Telescope photographs, which contains about 5,500 galaxies even within its smaller field of view. As a result, generated images of fake galaxies got a high level of fidelity with real samples from the XDF in terms of abundance, morphology, magnitude distributions, and colors. In this particular example, researchers have generated a 7.6-billion pixel 'generative deep field' spanning 1.45 degrees, showing that this approach can be extrapolated to other training sets for producing realistic pseudo surveys that can be successfully applied in astrophysics and other fields [13].

Despite some limitations of the proposed method (generated images are dependent on the training set; researchers couldn't reach stable learning output with more than three photometric bands) it also has undeniable advantages. The method is empirically driven because the data is used as the model, and it can be applied to generate extremely realistic artificial images for the design, development, and exploitation of new astronomical surveys. For instance, the technique allows assembling large training sets for different fractionalization and classification tasks in astrophysics [13]. The suggested generative technique makes it possible to expand small pieces of information retrieved from the early phases of a new survey to a level that will be applicable for training deep learning models. Described categorization and grouping algorithms can be effectively trained on the generated data and be implemented towards new data which can lead to expediting the processing of data of new surveys. Equation 4 represents a schematic architecture of the method [13]:

$$
D_{R}(x)=\left\{\begin{array}{l}
S\left(C(x)-\mathbb{E}_{x_{f} \sim Q} C\left(x_{f}\right)\right) \text { for real } x \\
S\left(C(x)-\mathbb{E}_{x_{r} \sim \mathbb{P}} C\left(x_{r}\right)\right) \text { for generated } x
\end{array},\right.
$$

where $S$ is the activation function (sigmoid), $x$ is data, $D_{R}(x)$ is the discriminator, $C(x)$ is the output of the final layer without activation function, $x_{f}$ stands for faked images, $x_{r}$ real images, $\mathbb{E}$ is an expectation.

\section{E. MORPHEUS - DL TOOL FOR ANAL YSIS OF ASTRONOMICAL IMAGES}

Surveying galaxies is a major instrument of observational astronomy. Ryan Hausen and Brant Robertson designed and described a deep learning framework for pixel-level analysis of astronomical image data - Morpheus [15]. This model helps astronomers to automatically classify galaxies by their shape or morphology. It implements deep learning methods in order to perform diverse astronomical tasks such as source detection, segmentation, and morphological classification which is carried out pixel-by-pixel through a semantic segmentation approach - a modified version of the computer vision algorithm.

Technically speaking, Morpheus is implemented as a convolutional neural network (Figure 3) similar to the $U$-Net framework and designed through a combination of Python 3 as the main tool and TensorFlow as a machine learning library. It is constructed from a series of so-called "blocks" that unify multiple reusable operations $[15,16]$.

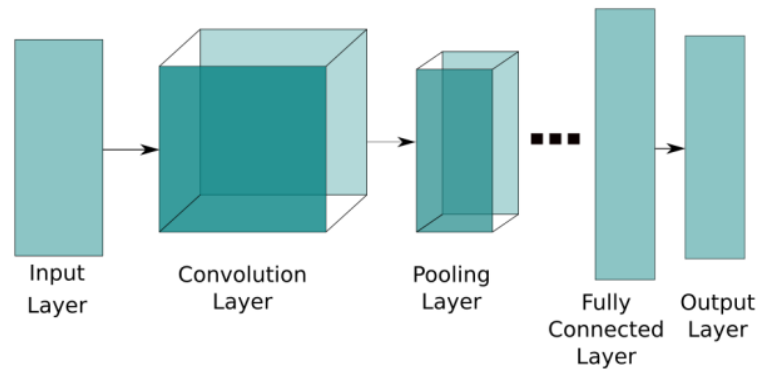

Figure 3. Convolutional neural network.

Represented by the Morpheus team pixel-by-pixel classification technique of astronomical images can be considered as an effective method of data analysis with wide applicability provided that suitable training datasets are available. The framework showed promising results with different datasets. As a performance assessment tool, CANDELS HLF and 3D-HST data were used, and Morpheus demonstrated a strong capability for morphological classification and object detection.

\section{F. GAN \& DEVELOPING NEW MOLECULES}

Zhavoronkov et al. described the process of developing new molecules that lasted only 21 days using artificial intelligence and GAN; the molecules have been successfully tested in mice. Customized and proprietary data were used 
as training and test datasets. The described approach has successfully passed experimental validation. The cost of the method is only a small fraction of the cost associated with the traditional approach to conventional drug discovery [17]. The newly developed deep generative model GENTRL (generative tensorial reinforcement learning) showed successful results in terms of de novo small-molecule design; it was applied as a system for discovering potent inhibitors of discoidin domain receptor 1 (DDR1), molecules that are involved in the regulation of cell functions and related to fibrosis and other diseases. It is anticipated that this method can be improved further as a perspective approach to identify drug candidates.

It was also reported that GANs are able to successfully design novel molecules for different inflammation-, fibrosis, and cancer-inducing protein targets [18]. During this work, two conditional GANs were stacked as one functional deep learning chain (conditional GANs and Wasserstein GAN with gradient penalty (WGAN-GP)) in order to achieve experimental expectations: the second network improved the results of the first one (stage 2 and stage 1). The following equations represent loss functions for the two stages mentioned above (stage 1 and stage 2 respectively, equations 5-8) [18]:

$$
\begin{gathered}
\mathcal{L}_{D_{0}}=\mathbb{E}_{x \sim p_{\text {real }}}\left[-D_{0}(x)\right] \\
+\mathbb{E}_{Z \sim p_{Z}, c \sim p_{\text {real }}}\left[D_{0}\left(G_{0}(z, c)\right)\right], \\
+\lambda \mathbb{E}_{\hat{x} \sim p_{x}}\left[\left(\left\|\nabla_{\hat{x}} D_{0}(\hat{x})_{2}-1\right\|\right)^{2}\right] \\
\mathcal{L}_{G_{0}}=\mathbb{E}_{Z \sim p_{z}, c \sim p_{\text {real }}} \\
{\left[-D_{0}\left(G_{0}(Z, c)\right)-\alpha \log \left(f_{0}\left(G_{0}(z, c), c\right)\right)\right],}
\end{gathered}
$$

where the generator is conditioned by a variable $c-G_{0}(z, c)$, $G$ and $D$ are generator and discriminator respectively, $z$ is a random noise vector sampled from $\left(p_{z}\right), x$ is data $(x$ and $c$ also represent a molecule representation and a gene expression signature), $p_{\text {real }}$ - real data distribution, $f_{0}$ is a function that represents a neural network and measures the probability of a gene expression signature, $\lambda$ and $\alpha$ are regularization parameters;

$$
\begin{gathered}
\mathcal{L}_{D_{1}}=\mathbb{E}_{x \sim p_{\text {real }}}\left[-D_{1}(x)\right] \\
+\mathbb{E}_{S_{0} \sim p_{G_{0}}, c \sim p_{\text {real }}}\left[D_{1}\left(G_{1}\left(s_{0}, c\right)\right)\right], \\
+\lambda \mathbb{E}_{\hat{x} \sim p_{\widehat{x}}}\left[\left(\left\|\nabla_{\hat{x}} D_{1}(\hat{x})\right\|_{2}-1\right)^{2}\right] \\
\mathcal{L}_{G_{1}}=\mathbb{E}_{S_{0} \sim p_{G_{0}}, c \sim p_{\text {real }}} \\
{\left[-D_{1}\left(G_{1}\left(s_{0}, c\right)\right)-\alpha \log \left(f_{1}\left(G_{1}\left(s_{0}, c\right), c\right)\right)\right],}
\end{gathered}
$$

where $\left(G_{1}\left(s_{0}, c\right)\right)$ is the generator, $\left(D_{1}(x)\right)$ is the discriminator, $G_{1}$ takes the output of $G_{0}\left(s_{0}=G_{0}(z, c)\right)$ and the gene expression signature $(c)$ as an input (instead of random noise).

\section{G. GANS IN DISCOVERING NEW MATERIALS \& PREDICTING CRYSTAL STRUCTURE}

The combined techniques of machine learning and GAN have confirmed their applied effectiveness in discovering new stable materials and predicting their crystal structure, which was described by Schmidt et al. [19]. A similar method called CrystalGAN was proposed by Asma Nouira et al., which made it possible to identify cross-domain connections in real data and to create new crystal structures. The proposed approach has demonstrated that it can efficiently integrate knowledge sets provided by human experts [20]. The GAN model showed its capability to generate new solid crystallographic structures.

CrystalGAN was able to successfully identify crossdomain connections in real data and generate novel structures. The model can be considered as the first GAN that has been explicitly designed to generate scientific data in materials science. CrystalGAN showed promising results and coped with the challenging task of discovering novel materials for hydrogen storage. Diverse GAN architectures are currently being studied in order to receive data of even higher complexity (compounds that consist of four or five chemical elements). It is important to stress that CrystalGAN as a general model that can be effectively adapted to any scientific task [20].

\section{H. DEEP LEARNING FOR DEEPFAKES CREATION AND DETECTION}

Deepfakes pose a serious threat to a person's personal safety since fabricated media data can be used to discredit a person by damaging their reputation (fake pornography, fake news, fraud, hoaxes, etc); they can provoke political instability, trigger violence, or even a war conflict.

Deepfakes are usually created with a special type of neural network that is called an autoencoder that studies effective information codings in an unsupervised way. Using the encoder-decoder chain the method allows replacing the face of one person with another (in video or photograph; for instance, Reface and DeepNude applications).

One of the powerful tools that enhance the capabilities of deepfakes is a generative adversarial network (GAN). A GAN trains a decoder (generator) and a discriminator in an adversarial interplay that makes fabricated data difficult to identify since two networks are consecutively evolving. As soon as the deepfake is identified, the system will immediately correct the defect and its further detection will be difficult. Due to the increasing quality of deepfake samples, detection methods also need to be improved. It has been suggested to make a benchmark data set of deepfakes that will help in developing effective detection methods [21].

This should simplify the process of training detection algorithms that require a massive training set. To better understand the possible methods of countering fakes, it is highly important to thoroughly study GANs. One of the perspective methods suggests detecting deepfakes by analyzing convolutional traces [22]. The approach is based on examining digital "fingerprints" to discriminate generated images and distinguish them from real photographic data. 


\section{GANS IN BIOMEDICAL INFORMATICS}

Deep learning and GAN methods are actively used in medical imaging (X-ray radiography, magnetic resonance imaging, positron emission tomography, etc.) as a powerful tool that allows health-care experts and radiologists to detect serious medical conditions and diseases at early stages with a high percentage of accuracy.

Since image data for many diseases are scarce, there is an urgent need for additional sources of information for training models. When there are not enough images for model training, GANs can generate high-quality samples that can be successfully applied as a solution in medical image analysis [23]. CycleGAN utilizes a cycle sequence loss to ensure model learning without paired data.

Thus, it can map from one domain (A in Figure 4) to another (B in Figure 4) without pairwise alignments between the source and target domain [24].

Wolterink et al. [25] described an application of CycleGAN in the radiotherapy treatment planning to CT and MRI images of patients with brain tumors.

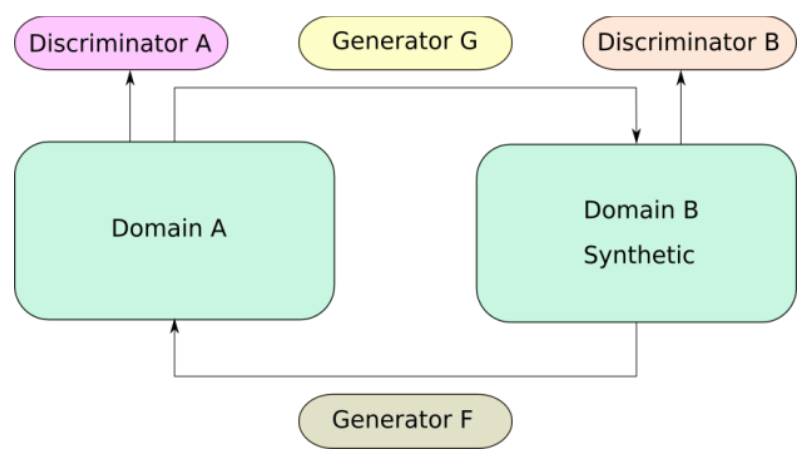

Figure 4. CycleGAN schematics.

Results demonstrate that Cycle-GAN outperforms a conventional single GAN trained with paired images. Li et al. [26] applied the GAN approach to predicting the possibility of whether a patient has a rare disease or not; the prediction accuracy was 5\% higher compared to standard methods. Recent studies also suggest that GANs can be engaged in solving the problem of lack of data in bioinformatics due to the capability of the network to generate high-quality data samples [27].

Models trained on small datasets demonstrate high bias, tend to overfit, and produce inaccurate predictions in terms of classification tasks.

\section{J. GANS FOR CREATING FONT EXAMPLES}

GAN can be also effective as a tool for creating new fonts and unique hand-written symbols like digits and letters. A standard GAN method in a combination with $A D A M$ optimizer was tested on the MNIST dataset. The experiment conducted by the authors showed the potential of the said approach to generate high-quality graphic samples of handwritten symbols. The overwhelming majority of obtained symbols looked similar to real digits, they were distinguishable by a sufficient level of clarity, structure, shape, and there was no significant graphic noise (Fig. 5) [28].

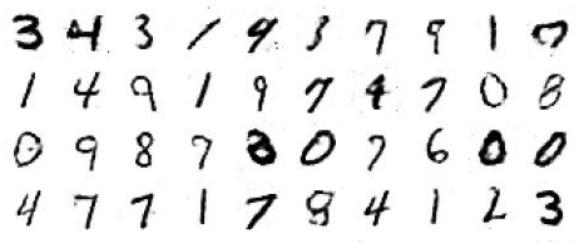

Figure 5. Samples generated by a GAN based on the MNIST dataset.

To obtain results of even higher quality the GAN parameters can be experimentally readjusted.

It should also be noted that a similar architecture can be used in typography to generate new font models, as well as in applied forensics to generate new training handwritten samples that can be used as part of datasets for training handwriting recognition and identification systems.

More examples can be reviewed and analyzed, but it is barely possible to list them all. The approaches reviewed above and summarized in Table 1 are practical confirmation of the high efficiency of using deep learning and generative adversarial neural networks in a wide range of applied areas in the context of sample and image data processing.

Alternatively, other generative models can be considered for the similar range of tasks:

- Variational Autoencoders (VAE) have density estimation, invertible, stable training, better diversity, but (a) the quality of the synthesized samples is much lower than GANs can produce and (b) slow learning speed.

- Autoregressive Model - more diverse samples, but learning requires supervision.

- Flow Models - lower quality samples.

- Hybrid Models - less stable.

GANs are still the best option when realistic generation is the main goal. 
Table 1. Peculiarities and possible modifications of the reviewed heuristics.

\begin{tabular}{|c|c|c|c|}
\hline Application Area & Technology/Models & Specificity & $\begin{array}{c}\text { Possible Modification } \\
\text { Methods }\end{array}$ \\
\hline Observational Astronomy & $\begin{array}{l}\text { GAN and DL for recovering } \\
\text { features in galaxy images } \\
\text { and photometric redshift } \\
\text { estimation; Fader network, } \\
\text { Spatial GAN (SGAN), } \\
\text { Morpheus. }\end{array}$ & $\begin{array}{l}\text { An important component of } \\
\text { cosmological research. } \\
\text { Limited capacity of the } \\
\text { training sets; domain know- } \\
\text { ledge oriented; generated } \\
\text { image samples depend on } \\
\text { the training datasets. }\end{array}$ & $\begin{array}{l}\text { Applying synthesized } \\
\text { samples for enlarging } \\
\text { existing data-sets; dropout } \\
\text { and random forest methods } \\
\text { to avoid overfitting; } \\
\text { architecture optimization. }\end{array}$ \\
\hline Health Care/Longevity & $\begin{array}{l}\text { DL and GAN; Generative } \\
\text { Tensorial Reinforcement } \\
\text { Learning (GENTRL); } \\
\text { Wasserstein GAN (WGAN). }\end{array}$ & $\begin{array}{l}\text { A highly effective approach } \\
\text { with promising results; } \\
\text { further applications of the } \\
\text { method and its limitations } \\
\text { are being actively studied. }\end{array}$ & $\begin{array}{l}\text { Potentially more effective } \\
\text { combinations of models re- } \\
\text { quire further research. }\end{array}$ \\
\hline Materials Science & $\begin{array}{l}\text { ML/DL; GAN; } \quad \text { Crystal- } \\
\text { GAN. }\end{array}$ & $\begin{array}{l}\text { A successful approach for } \\
\text { the design of new materials. }\end{array}$ & $\begin{array}{l}\text { Cross-domain knowledge } \\
\text { bases in physics and chem- } \\
\text { istry are needed for further } \\
\text { optimization and designing } \\
\text { even more productive } \\
\text { models. }\end{array}$ \\
\hline Deep Fake & $\begin{array}{l}\text { Standard GANs, autoenco- } \\
\text { der network. }\end{array}$ & $\begin{array}{l}\text { A high degree of influence } \\
\text { on the social factor and pub- } \\
\text { lic safety. }\end{array}$ & $\begin{array}{l}\text { Further improvement of } \\
\text { detection methods; creation } \\
\text { of a benchmark data set of } \\
\text { deepfakes; convolutional } \\
\text { trace identification appro- } \\
\text { aches. }\end{array}$ \\
\hline Bioinformatics & DL and GAN; CycleGAN. & $\begin{array}{l}\text { The method maps one } \\
\text { domain to another without } \\
\text { pairwise alignments bet- } \\
\text { ween the source and target } \\
\text { do-main. The suggested } \\
\text { approach can help to solve } \\
\text { the problem of lack of data } \\
\text { in bioinformatics. }\end{array}$ & $\begin{array}{l}\text { Optimizing the architecture, } \\
\text { examining the possibility of } \\
\text { adding Gaussian noise bet- } \\
\text { ween loops and analyzing its } \\
\text { impact on system perfor- } \\
\text { mance. }\end{array}$ \\
\hline $\begin{array}{c}\text { Typography and } \\
\text { Handwritten Samples }\end{array}$ & $\begin{array}{l}\text { GAN; Deep Convolution } \\
\text { GANs. }\end{array}$ & $\begin{array}{l}\text { Requires high-quality data- } \\
\text { sets and sophisticated algo- } \\
\text { rithmic optimization me- } \\
\text { thods. }\end{array}$ & $\begin{array}{l}\text { Network parameters re- } \\
\text { adjustment; normalizing a } \\
\text { number of training epochs; } \\
\text { applying additional combi- } \\
\text { nations of optimization } \\
\text { techniques. }\end{array}$ \\
\hline
\end{tabular}

\section{ADVANCED APPROACHES FOR IMPROVING DEEP LEARNING AND GANS APPLIED EFFICIENCY}

Given the above descriptions, there are several possible ways that could help to improve the effectiveness of all these approaches for various scientific fields.

\section{A. IMPROVING PERFORMANCE USING DATA}

As for improving performance with data, it needs to be stressed that the more training data collected, the better the performance since this directly affects the quality of the deep learning models used. The efficiency of any algorithm loses its value if the amount of data is insufficient for full-fledged training.

If there is not enough data in the training set, it is advisable to consider artificial data generation as an option. As it was mentioned by Schawinski et al. [7], the main constraint of their approach was the limited capacity of the available training set. Fabricated images generated by GANs may be considered as a possible solution for this issue. In other words, special GAN architectures can assist other GANs to improve their performance and results. When it comes to image data, we can either synthesize new images or randomly modify samples of existing images; we can also use random rotation or shifting images or adding simulated noise. The principle of data augmentation applies to other data types as well (vectors of numbers, text, etc).

Lack of training data can result in overfitting, thus the best way to avoid it is to provide a deep neural network with increased quantity of quality training data. Generative modeling with GANs can fill the gap by reinforcing smaller datasets with new synthesized high-quality images. The deep convolutional GAN architecture (DCGAN) is capable of creating photorealistic graphical samples that accurately 
correspond to the feature distributions of real galaxies in terms of statistical estimation [29]. It is worth noticing, though, that these are limited by the accuracy of the probability density estimates.

Levi Fussell and Ben Moews experimentally proved that StackGAN can be applied as a second-stage architecture and form a combination system with DCGAN in order to synthesize fabricated galaxy images with higher resolutions, avoiding the obstructions that DCGAN models experience with such resolutions [30, 31].

Data rescaling in the context of the applied activation functions also plays an important role. It's sometimes useful to normalize data values and rescale them; between 0 and 1 if it comes to sigmoid activation functions, between 0 and infinity if it's rectified linear unit (ReLU), -1 and 1 if it's hyperbolic tangent (tanh), for instance. This rescaling principle can be applied to other activation functions as well.

\section{B. ALGORITHMIC APPROACH}

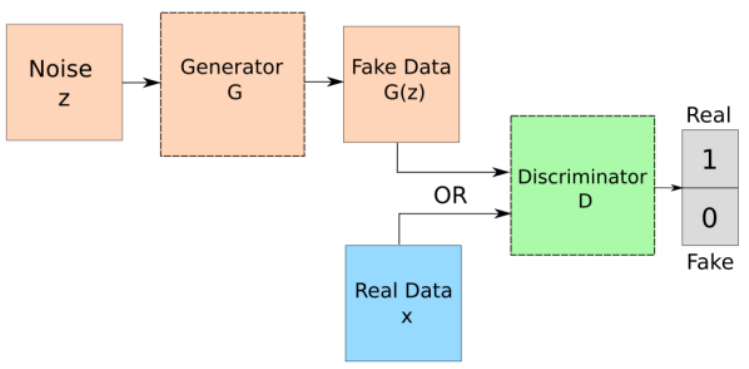

Figure 6. Structure of interactions between Generator and Discriminator networks in GAN.

As for the algorithmic approach, if GANs mainly use the original algorithm designed by Goodfellow et al. (Figure 6) and its modifications, other deep learning algorithms use diverse models and approaches that vary from case to case [9].

Equation 9 represents a mathematical description of GAN as a variation of the minimax two-player game [9]:

$$
\begin{gathered}
\min _{G} \max _{D} V(D, G)=\mathbb{E}_{\boldsymbol{x} \sim p_{\text {data }}(\boldsymbol{x})}[\log D(\boldsymbol{x})]+ \\
\mathbb{E}_{\mathbf{z} \sim p_{\mathbf{z}}(\mathbf{z})}[\log (1-D(G(\mathbf{z})))],
\end{gathered}
$$

where $G$ is the generator network; $D$ is the discriminator network; $x$ is a sample of real data; $p_{z}(z)$ - is a prior on input noise variables; $z$ is noise; $D(x)$ is the probability that $x$ actually is from the data rather than from the generator; $G(z)$ is the generator output; $\mathbb{E}$ is prediction (expectation), the first part represents the discriminator's predictions on the real data, the second one - the discriminator's predictions on the fake/generated data; $V(D, G)$ is the value function of discriminator and generator in the two-player minimax game.

Each time proper algorithmic diagnostics should be applied to the desired deep learning model. All examples reviewed in the paper can be experimentally modified in terms of weight configuration, network topology, types of activation functions, learning rate, batches, and number of epochs. The network topology depends on the task at hand. Since at the moment it is generally accepted that there are no unified rules regarding how many layers or how many neurons are needed for a particular configuration of a neural network, these parameters are selected experimentally.

\section{HYBRID METHODS AND OPTIMIZATION}

The new technique proposed by Karras et al. allows training generative adversarial networks with limited data. They have suggested methods of adaptive discriminator augmentation in GANs and described the following overfitting heuristics [32]:

$$
r_{v}=\frac{\mathbb{E}\left[D_{\text {train }}\right]-\mathbb{E}\left[D_{\text {validation }}\right]}{\mathbb{E}\left[D_{\text {train }}\right]-\mathbb{E}\left[D_{\text {generated }}\right]} \quad r_{t}=\mathbb{E}\left[\operatorname{sign}\left(D_{\text {train }}\right)\right],
$$

where the discriminator outputs are denoted by $D_{\text {train }}$ for the training set, $D_{\text {validation }}$ for the validation set, and $D_{\text {generated }}$ for the generated images, $\mathbb{E}$ denotes their mean over $N$ consecutive minibatches. During the experiment, $N$ was equal to 4 , which corresponds to $4 \times 64=256$ images. For both parts of heuristics, $r=0$ stands for no overfitting and $r=1$ implies complete overfitting. The goal of the experiment was to adjust the augmentation probability $p$ so that the selected heuristic fits an appropriate target value. Two heuristic units: $r_{v}$ signifies the output for a validation set relative to the training set and generated images, $r_{t}$ evaluates the fraction of the training set that gets positive discriminator outputs [32]. Additional augmentation and regularization approaches in the GAN context have been suggested by Cubuk et al. (September 2019) and Zhang et al. (February 2020) [33, 34].

Recent research shows that a combination of multiple GANs can create generated data with higher quality compared to a conventional single GAN [32]. It should also be noted that sometimes it happens that due to oversimplified loss function GANs do not learn the way they are expected to (mode collapse, vanishing gradients, convergence). This problem remains one of the active areas of research at the moment.

Sophisticated regularization methods, such as dropout (equation 11), can also help to avoid overfitting in neural networks [35]:

$$
\widehat{\mathrm{w}}_{j}=\left\{\begin{array}{ll}
\mathrm{w}_{j}, & \text { with } P(c) \\
0, & \text { otherwise }
\end{array},\right.
$$

where $\mathrm{P}(\mathrm{c})$ is the probability of $\mathrm{c}$ (stands for "keeping a weight" factor) to keep a row in the weight matrix, $\mathrm{w}_{j}$ is a real row in the weight matrix before dropout, $\widehat{w}_{j}$ is a diluted row in the weight matrix. Dropout randomly omits (or "drops out") neurons of a neural network (both hidden and visible) during the training process. It should be noted that zeroing out the node does not impact the end result.

$K$-fold cross-validation is another method that can be applied to a model in order to avoid overfitting. Data is 
separated into $\mathrm{K}$ randomly-assigned fragments where one fragment is earmarked as test data. The remaining combined K-1 (minus one) fragments are used for training and after that, results need to be evaluated with the test set. This cycle then reiterates for each fragment and the average of the $\mathrm{K} \mathrm{r}$ squared scores is calculated, or the results displayed in a box plot to give the median value and identify outliers.

Another possible way to improve the results obtained using deep learning methods is algorithmic model rotation. To solve the same problem, completely different types of neural networks and methods of their modification can be applied. Restarts indicate the impact of local minima and saddle points in the loss function.

Experimenting with linear and non-linear methods (logistic regression, polynomial regression, and multiple regression) along with tree methods like gradient boosting, classification and regression trees, and random forest decreases variance and could give different results in terms of productivity. For classification and regression analysis in neural networks, support-vector networks and k-nearest neighbors algorithms also demonstrate high-level efficiency. Sometimes, to obtain more efficient results, it makes sense to resort to the hybridization of models, combining the following approaches with each other: learning vector quantization, Boltzmann machines, multilayer perceptron, convolutional neural network, long short-term memory architecture, competitive networks such as GANs, autoencoder networks, deep stacking networks. The list of architectures given is only illustrative since the number of all possible combinations increases exponentially.

Performance can be also improved by involving algorithms for gradient-based optimization such as RMSProp, AdaGrad, Momentum, Adagrad, Adadelta, and ADAM. Upon experimental evaluation, $A D A M$ demonstrated strong results with logistic regression, multilayer neural networks, convolutional neural networks, and performed equal or better than RMSProp, regardless of hyper-parameter settings [36]. Using ADAM as an example, and considering it takes its name from "adaptive moment estimation," we can see that this method utilizes evaluations of first and second moments of gradient adjusting the learning rate for each weight parameter of the neural network. The moment is a numerical characteristic of the distribution of a given random variable (expected value of the variable to the $n$-th power, equation 12 ):

$$
M_{n}=E\left[V_{\text {ran. }}^{n}\right] \text {, }
$$

where $M$ is the moment, $E$ is the expected value of the variable, $V_{\text {ran }}$ is a random variable.

Deep learning optimization models are still an open domain and still require in-depth research, both in terms of mathematical groundings and in terms of software and hardware implementation.

\section{CONCLUSIONS}

Since the amount of information is constantly increasing, scientists need modern and efficient tools to examine and analyze the data they receive. Nowadays researchers have unprecedented access to advanced AI tools for gathering, retrieving, processing, and recovering images and statistical data $[37,38,39]$. It is certain that machine learning can process and analyze information much faster than humans or other computational methods, furthermore, it can comprehend data patterns and liaisons that we do not even recognize, e.g., it may detect diverse types of galaxies before scientists know they exist.

In the article, the authors analyze the types of modern GAN architectures and existing approaches to their design, as well as the main advantages and prospects for widespread implementation of GAN and deep learning (DL) for solving topical problems of artificial intelligence. The above analysis of successful cases illustrates and confirms the high efficiency of GANs and DL in astronomy, molecular biology, materials science, bioinformatics, handwriting recognition, and deepfake detection. The authors provide an analysis and offer proposals for the development of advanced approaches in terms of design and implementation of GANs and DL. In the future, the authors plan to develop software for the implementation of GAN and DL based on the discussed advanced approaches.

Processing instrumental images using artificial neural networks can accelerate further research and help in reconstructing imaging data even for nonstandard and unstudied phenomena. GANs can effectively remove noise and provide as clear of an image as possible due to their ability to recover graphical data that have damaged or missing pixels, or unwanted instrumental artifacts. Thus, the machine learning methods and GANs should be considered as the most promising assistive technologies for science as a whole.

GAN is one of the relatively new DL technologies (2014), requires a thorough research and analysis, significantly affects various aspects of scientific and technological development (creation of new drugs, space exploration) and socio-political life including Deep Fake problem (photo, video, audio).

As for drawbacks and limitations, it is worth noticing that in order to train a machine learning system we need a lot of labeled and preprocessed information. Moreover, until recently, the scientific community simply had no data about some substantial aspects that are important for preparing effective training sets. In addition, neural networks are being considered as a kind of black box: researchers do not always understand exactly how artificial neural networks operate, especially when it comes to complex architectures with many hidden layers of neurons. Using tools without a proper level of understanding of how they work is a matter of concern among scientists.

Nevertheless, DL systems continue contributing to progress across a range of different scientific fields [40, 41], and therefore, the prospects for the further use of machine learning in applied scientific research, as well as methods for its improving and optimizing, should continue to be 
comprehensively studied. Proper technological unification and combination of research efforts can lead to revolutionary results.

The main task of the article is an in-depth scientific analysis of current practical approaches in terms of application of deep learning methods, including generative models, in the most important spheres of human activity, because today DL and GANs still need thorough study and research: theoretical basis, areas and limits of application, security and safety. Awareness of the scientific community in modern methods of artificial intelligence and the need for access to pervasive analytical materials are among the key aspects influencing the speed, intensity, and novelty of research. The article analyzes innovative approaches in DL and GANs, provides an integral assessment of their effectiveness, and offers practical and theoretical suggestions for improvement.

It is expected that modern deep learning technologies will make a significant contribution to science and the development of research methodology, provided that proper convergence between the vast majority of scientific fields is achieved.

\section{References}

[1] S. Russell, P. Norvig, Artificial Intelligence: A Modern Approach, 3rd ed., Upper Saddle River, New Jersey: Prentice Hall, 2009, 1 p.

[2] P. Barmby, Astronomical observations: a guide for allied researchers, 2019, [Online]. Available at: https://arxiv.org/abs/1812.07963, https://doi.org/10.21105/astro.1812.07963.

[3] E. C. Sutton, Observational Astronomy. Techniques and Instrumentation, Cambridge University Press, 2011, 1 p.

[4] J. Lee, P. L. Freddolino, Y. Zhang, Ab initio protein structure prediction, D.J. Rigden (ed.), From Protein Structure to Function with Bioinformatics, 2017, pp. 1-33. https://doi.org/10.1007/978-94-0241069-3_1.

[5] DeepMind, "AlphaFold: Using AI for scientific discovery," Nature, vol. 577, pp. 706-710, 2020.

[6] S. Roberts, A. McQuillan, S. Reece, S. Aigrain, "Astrophysically robust systematics removal using variational inference: application to the first month of Kepler data," Monthly Notices of the Royal Astronomical Society, vol. 435, pp. 3639-3653, 2013. https://doi.org/10.1093/mnras/stt1555.

[7] K. Schawinski, C. Zhang, H. Zhang, L. Fowler, G. K. Santhanam, "Generative adversarial networks recover features in astrophysical images of galaxies beyond the deconvolution limit," Monthly Notices of the Royal Astronomical Society: Letters, vol. 467, issue 1, pp. 110114, 2017. https://doi.org/10.1093/mnrasl/slx008.

[8] P. Magain, F. Courbin, S. Sohy, "Deconvolution with Correct Sampling," The Astrophysical Journal, pp. 472-477, 1998. https://doi.org/10.1086/305187.

[9] I. Goodfellow, J. Pouget-Abadie, M. Mirza, B. Xu, D. Warde-Farley, S. Ozair, A. Courville, J. Bengio, "Generative Adversarial Networks," Proceedings of the International Conference on Neural Information Processing Systems (NIPS), 2014, pp. 2672-2680.

[10] A. D'Isanto, K. L. Polsterer, "Photometric redshift estimation via deep learning. Generalized and pre-classification-less, image based, fully probabilistic redshifts," Astronomy \& Astrophysics, vol. 609, A111, pp. 1-16, 2018. https://doi.org/10.1051/0004-6361/201731326.

[11] G. Lample, N. Zeghidour, N. Usunier, A. Bordes, L. Denoyer, M. Ranzato, "Fader networks: Manipulating images by sliding attributes," Proceedings of 31st Conference on Neural Information Processing Systems, USA, 2017, pp. 1-10.

[12] K. Schawinski, M. D. Turp, C. Zhang, "Exploring galaxy evolution with generative models," Astronomy \& Astrophysics, vol. 616, L16, pp. 1-4, 2018. https://doi.org/10.1051/0004-6361/201833800.
[13] M. J. Smith, J. E. Geach, "Generative deep fields: arbitrarily sized, random synthetic astronomical images through deep learning," Monthly Notices of the Royal Astronomical Society, vol. 490, issue 4, pp. 4985-4990, 2019. https://doi.org/10.1093/mnras/stz2886.

[14] N. Jetchev, U. Bergmann, R. Vollgraf, "Texture Synthesis with Spatial Generative Adversarial Networks," Proceedings of Workshop on Adversarial Training, NIPS '2016, Barcelona, Spain, 2016, pp. 1-11.

[15] R. Hausen, B. Robertson, Morpheus: A Deep Learning Framework for Pixel-Level Analysis of Astronomical Image Data, 2019, [Online] Available at: https://arxiv.org/abs/1906.11248

[16] O. Ronneberger, P. Fischer, T. Brox, "U-Net: Convolutional networks for biomedical image segmentation," Proceedings of the 18th International Conference Medical Image Computing and ComputerAssisted Intervention, Munich, Germany, October 5-9, 2015, pp. 1-8.

[17] A. Zhavoronkov, Y. A. Ivanenkov, A. Aliper et al., "Deep learning enables rapid identification of potent DDR1 kinase inhibitors," Nature Biotechnology, vol. 37, pp. 1038-1040, 2019. https://doi.org/10.1038/s41587-019-0224-x.

[18] O. Méndez-Lucio, B. Baillif, D.-A. Clevert, D. Rouquié, J. Wichard, De novo generation of hit-like molecules from gene expression signatures using artificial intelligence, 2020. https://doi.org/10.1038/s41467-019-13807-w.

[19] J. Schmidt, M. R. G. Marques, S. Botti et al., Recent advances and applications of machine learning in solid-state materials science, 2019. https://doi.org/10.1038/s41524-019-0221-0.

[20] A. Nouira, N. Sokolovska, J.-C. Crivello, "CrystalGAN: Learning to discover crystallographic structures with generative adversarial networks," Proceedings of the AAAI Spring Symposium: Combining Machine Learning with Knowledge Engineering, Stanford University, USA, March 25-27, 2019, pp. 1-9.

[21] T. T. Nguyen, C. M. Nguyen, D. T. Nguyen, D. T. Nguyen, S. Nahavandi, Deep Learning for Deepfakes Creation and Detection: A Survey, 2020, [Online]. Available at: https://arxiv.org/abs/1909.11573

[22] L. Guarnera, O. Giudice, S. Battiato, "DeepFake detection by analyzing convolutional traces," Proceedings of the IEEE Conference on Computer Vision and Pattern Recognition Workshops, 2020, pp. 1-10. https://doi.org/10.1109/CVPRW50498.2020.00341.

[23] L. Lan, L. You, Z. Zhang, Z. Fan, W. Zhao, N. Zeng, Y. Chen, X. Zhou, Generative Adversarial Networks and its Applications in Biomedical Informatics, https://doi.org/10.3389/fpubh.2020.00164.

[24] J.-Y. Zhu, T. Park, P. Isola, A. A. Efros, Unpaired Image-to-Image Translation using Cycle-Consistent Adversarial Networks, 2020, [Online]. Available at: https://arxiv.org/abs/1703.10593

[25] J. M. Wolterink et al., Deep MR to CT Synthesis Using Unpaired Data, in Tsaftaris S., Gooya A., Frangi A., Prince J. (eds) Simulation and Synthesis in Medical Imaging., SASHIMI 2017, Lecture Notes in Computer Science, 2017, vol. 10557, Springer, Cham, pp. 14-23.

[26] W. Li, Y. Wang, Y. Cai, C. Arnold, E. Zhao, Y. Yuan, Semisupervised Rare Disease Detection Using Generative Adversarial Network, 2018, [Online]. Available at: https://arxiv.org/abs/1812.00547

[27] M. Marouf, P. Machart, V. Bansal, C. Kilian, D. S. Magruder, C. F. Krebs, S. Bonn, Realistic in silico generation and augmentation of single-cell RNA-seq data using generative adversarial networks, 2020. https://doi.org/10.1038/s41467-019-14018-z.

[28] O. Striuk, Y. Kondratenko, I. Sidenko, A. Vorobyova, "Generative adversarial neural network for creating photorealistic images," Proceedings of the 2020 IEEE 2nd International Conference on Advanced Trends in Information Theory, Kyiv, Ukraine, November 27, 2020, pp. 1-4.

[29] M. C. Chan, J. P. Stott, "Deep-CEE I: Fishing for galaxy clusters with deep neural nets," Monthly Notices of the Royal Astronomical Society, $\begin{array}{llll}\text { vol. } 490, & \text { pp. } & 5770-5787, & \end{array}$ https://doi.org/10.1093/mnras/stz2936.

[30] Z. L. Wen, J. L. Han, F. S. Liu, "A Catalog of 132,684 clusters of galaxies identified from sloan digital sky survey III," The Astrophysical Journal Supplement, vol. 199, issue 2, article id. 34, pp. 1-12, 2012. https://doi.org/10.1088/0067-0049/199/2/34.

[31] L. Fussell, B. Moews, "Forging new worlds: high-resolution synthetic galaxies with chained generative adversarial networks," Monthly 
Notices of the Royal Astronomical Society, vol. 485, issue 3, pp. 32033214, 2019. https://doi.org/10.1093/mnras/stz602.

[32] T. Karras, M. Aittala, J. Hellsten, S. Laine, J. Lehtinen, T. Aila, "Training generative adversarial networks with limited data," Proceedings of the 34th Conference on Neural Information Processing Systems (NeurIPS 2020), Vancouver, Canada, June 2020, pp. 1-37.

[33] H. Zhang, T. Xu, H. Li, S. Zhang, X. Wang, X. Huang, D. Metaxas, "StackGAN: Text to photo-realistic image synthesis with stacked generative adversarial networks," Proceedings of the 2017 IEEE International Conference on Computer Vision (ICCV), Venice, Italy, 2017, pp. 5908-5916. https://doi.org/10.1109/ICCV.2017.629.

[34] E. D. Cubuk, B. Zoph, J. Shlens, Q. V. Le, RandAugment: Practical automated data augmentation with a reduced search space, 2019, [Online]. Available at: https://arxiv.org/abs/1909.13719. https://doi.org/10.1109/CVPRW50498.2020.00359.

[35] N. Srivastava, G. Hinton, A. Krizhevsky, I. Sutskever, R. Salakhutdinov, "Dropout: A Simple Way to Prevent Neural Networks from Overfitting," Journal of Machine Learning Research, vol. 15, pp. 1929-1958, 2014.

[36] D. P. Kingma, J. L. Ba, "Adam: A method for stochastic optimization," Proceedings of the 3rd International Conference for Learning Representations, San Diego, USA, 2015, pp. 1-15.

[37] R. Leizerovych, G. Kondratenko, I. Sidenko, Y. Kondratenko, "IoTcomplex for monitoring and analysis of motor highway condition using artificial neural networks," Proceedings of the 2020 IEEE 11th International Conference on Dependable Systems, Services and Technologies, DESSERT 2020, Kyiv; Ukraine; 14-18 May, 2020; Article No. 9125004, pp. 207-212. https://doi.org/10.1109/DESSERT50317.2020.9125004.

[38] K. Ivanova, G. Kondratenko, I. Sidenko, Y. Kondratenko, "Artificial intelligence in automated system for web-interfaces visual testing," CEUR Workshop Proceedings, vol. 2604, 2020, 4th International Conference on Computational Linguistics and Intelligent Systems, COLINS 2020; Lviv; Ukraine; 2020; pp. 1019-1031.

[39] V. M. Kuntsevich et al. (Eds), Control Systems: Theory and Applications. Series in Automation, Control and Robotics, River Publishers, 2018, $146 \mathrm{p}$.

[40] Y. Kondratenko, D. Simon, Structural and parametric optimization of fuzzy control and decision making systems, In: Zadeh L., Yager R., Shahbazova S., Reformat M., Kreinovich V. (eds), Recent Developments and the New Direction in Soft-Computing Foundations and Applications. Studies in Fuzziness and Soft Computing, Springer, Cham., vol. 361, 2018, pp. 273-289.
[41] Z. Gomolka, E. Dudek-Dyduch, Y. P. Kondratenko, "From homogeneous network to neural nets with fractional derivative mechanism," Proceedings of the International Conference on Artificial Intelligence and Soft Computing, ICAISC-2017, Rutkowski, L. et al. (Eds), Part I, Zakopane, Poland, 11-15 June, 2017, LNAI 10245, Springer, Cham, 2017, pp. 52-63. https://doi.org/10.1007/9783-319-59063-9 5 .

[42] J. Brownlee. 18 Impressive Applications of Generative Adversarial Networks (GANs), 2019, [Online]. Available at: https://machinelearningmastery.com/impressive-applications-ofgenerative-adversarial-networks/

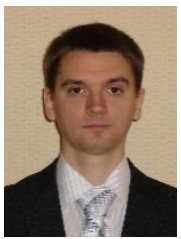

OLEKSANDR STRIUK, Ph.D. student and researcher at Petro Mohyla Black Sea National University (PMBSNU). Master of Science in System Analysis. Research interests include AGI, artificial neural networks, reasoning in $A l$, statistics, and probability theory.

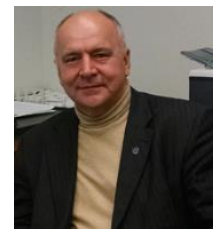

YURIY KONDRATENKO, Doctor of Science, Professor, Honour Inventor of Ukraine (2008), Corr. Academician of Royal Academy of Doctors (Barce-lona, Spain), Head of the Department of Intelligent Information Systems at Petro Mohyla Black Sea National University (PMBSNU), Ukraine. He has received (a) the Ph.D. (1983) and Dr.Sc. (1994) in Elements and Devices of Computer and Control Systems from Odessa National Polytechnic University, (b) several international grants and scholarships for conducting research at Institute of Automation of Chongqing University, P.R.China (1988-1989), Ruhr-University Bochum, Germany (2000, 2010), Nazareth College and Cleveland State University, USA (2003), (c) Fulbright Scholarship for researching in USA (2015/2016) at the Dept. of Electrical Engineering and Computer Science in Cleveland State University. Research interests include robotics, automation, sensors and control systems, intelligent decision support systems, fuzzy logic. 